\title{
BMJ Open HABIT+tDCS: a study protocol of a randomised controlled trial (RCT) investigating the synergistic efficacy of hand-arm bimanual intensive therapy (HABIT) plus targeted non-invasive brain stimulation to improve upper extremity function in school-age children with unilateral cerebral palsy
}

Andrew M Gordon (D , ${ }^{1}$ Claudio L Ferre, ${ }^{2,3}$ Maxime T. Robert, ${ }^{4}$ Karen Chin, ${ }^{1,2}$ Marina Brandao, ${ }^{5}$ Kathleen M Friel $^{2}$

To cite: Gordon AM, Ferre $\mathrm{CL}$, Robert MT, et al. HABIT+tDCS: a study protocol of a randomised controlled trial (RCT) investigating the synergistic efficacy of hand-arm bimanual intensive therapy (HABIT) plus targeted non-invasive brain stimulation to improve upper extremity function in schoolage children with unilateral cerebral palsy. BMJ Open 2022;12:e052409. doi:10.1136/ bmjopen-2021-052409

- Prepublication history and additional supplemental materia for this paper are available online. To view these files, please visit the journal online (http://dx.doi.org/10.1136/ bmjopen-2021-052409)

Received 14 April 2021 Accepted 27 January 2022

Check for updates

(C) Author(s) (or their employer(s)) 2022. Re-use permitted under CC BY-NC. No commercial re-use. See rights and permissions. Published by BMJ.

For numbered affiliations see end of article.

Correspondence to Professor Andrew M Gordon; ag275@columbia.edu

\section{ABSTRACT}

Introduction Unilateral spastic cerebral palsy (USCP) is characterised by movement deficits primarily on one body side. The best available upper extremity (UE) therapies are costly and intensive. Thus, there is an urgent need for better, more efficient and thus more accessible therapies. Transcranial direct current stimulation (tDCS) is non-invasive and may enhance physical rehabilitation approaches. The aim of this study is to determine whether tDCS targeted to the hemisphere with corticospinal tract (CST) connectivity enhances the efficacy of UE training in children with USCP. Our central hypothesis is that hand-arm bimanual intensive therapy (HABIT) combined with a tDCS montage targeting the hemisphere with CST connectivity to the impaired UE muscles will improve UE function more than HABIT plus sham stimulation. We will test this by conducting a randomised clinical trial with clinical and motor cortex physiology outcomes.

Methods and analyses 81 children, aged $6-17$ years, will be randomised to receive $2 \mathrm{~mA}$ anodal tDCS targeted to the affected UE motor map, $2 \mathrm{~mA}$ cathodal tDCS to the contralesional motor cortex or sham tDCS during the first 20 min of each HABIT session (10 hours: 2 hours/day for 5 days). Primary outcomes will be Box and Blocks Test, Assisting Hand Assessment and motor cortex excitability, determined with single-pulse transcranial magnetic stimulation. Secondary outcomes include ABILHAND-Kids, Canadian Occupational Performance Measure, Cooper Stereognosis, Dimension of Mastery Questionnaire and Participation and Environment Measure-Children and Youth. All measures will be collected before, immediately and 6 months after treatment. A group $\times$ test session Analysis of Variance will test differences among groups on all measures.

Ethics and dissemination The study has been approved by the BRANY Institutional Review Board (\#18-10-

\section{Strengths and limitations of this study}

- This study will determine whether targeting transcranial direct current stimulation (tDCS) based on corticospinal tract (CST) connectivity has a synergistic effect with bimanual training in children with unilateral spastic cerebral palsy (USCP).

- The approach will identify which targeted tDCS strategies lead to strongest expansion of motor maps.

- The study will dissect the interactions between CST laterality, non-invasive brain stimulation and motor training in children with USCP and examine them using an integrated approach.

- We do not expect to obtain equal sample sizes for each CST connectivity pattern, and may not be able to fully determine interactions if a group is underpowered.

- It is possible that anodal and cathodal tDCS polarity affects cortical excitability differently in children with USCP than their typical effects in adults.

285-512). We will leverage our subject and family relationships to maximise dissemination and share results with the academic and patient/family advocacy groups. Trial registration number NCT03402854.

\section{INTRODUCTION}

Background

Unilateral spastic cerebral palsy (USCP) is characterised by movement deficits, particularly upper extremity (UE) impairments, on one side of the body. Although significant strides to develop rehabilitation approaches to improve UE function in children with 
USCP have occurred, ${ }^{1}$ the best available UE therapies result in limited improvements in UE function, are costly and require large amounts of treatment time (ie, 4-6 hours/day for 2-3 weeks). Few families, particularly families of lower socioeconomic status, have the ability to engage their children in these intensive therapies ${ }^{23}$ and the time required to achieve the high intensity is a limiting factor for more widespread implementation. ${ }^{4}$ There is an urgent need for therapies that can deliver enhanced improvements but are more cost efficient and accessible.

Transcranial direct current stimulation (tDCS) is a noninvasive, low-risk method of delivering low levels of energy to the brain via saline-soaked sponge electrodes placed over the scalp. tDCS is portable, affordable and well tolerated in paediatric populations, making it an ideal strategy to combine with UE training. ${ }^{5}$ Typically, neurons stimulated by the anodal electrode are depolarised, whereas neurons stimulated by the cathodal electrode are hyperpolarised. ${ }^{6}$ Repeated sessions of motor training of desired motor behaviours with concurrent anodal tDCS targeted to motor cortex of healthy adults facilitates learning through enhancement or consolidation. ${ }^{7}$

A consensus group in neurology ${ }^{8}$ hypothesised that tDCS may increase the rate of motor learning in healthy adults. Enhanced motor learning was seen in typically developing children following contralateral anodal tDCS stimulation. ${ }^{9} 10$ A recent meta-analysis in adults with stroke suggested that tDCS was beneficial in improving activities of daily living, with contralesional cathodal stimulation likely targeting impaired interhemispheric inhibition (IHI) being most effective. ${ }^{11}$ Others have questioned whether IHI is the main driver of impaired UE function. ${ }^{12}$ Although promising, the results of tDCS studies in limited samples of children with USCP have yielded equivocal differences between stimulation and sham groups, ${ }^{13-17}$ possibly due to underdosing the tDCS and overdosing the paired motor skill training, with the latter washing out the additional effects of tDCS. Furthermore, tDCS studies have not specifically targeted the motor map of the affected UE. Thus, the montage may result in different outcomes depending on whether the lesioned hemisphere maintains contralateral corticospinal tract (CST) connectivity to the affected hand's muscles or the connectivity pattern has been reorganised, with the contralesional hemisphere controlling both hands (figure 1).

\section{Objectives}

The overall objective of this project is to determine how to optimally target tDCS to enhance the efficacy of UE training in children with USCP. Our central hypothesis is that combined hand-arm bimanual intensive therapy (HABIT) and an individualised tDCS montage targeting the hemisphere with CST connectivity to the impaired UE muscles will improve UE function more than HABIT plus sham stimulation. We will also determine interactions between HABIT +tDCSand motor cortex physiology. We hypothesise that children who receive anodal tDCS targeted to the individual's hemisphere containing CST

\section{Targeted tDCS Untargeted tDCS}
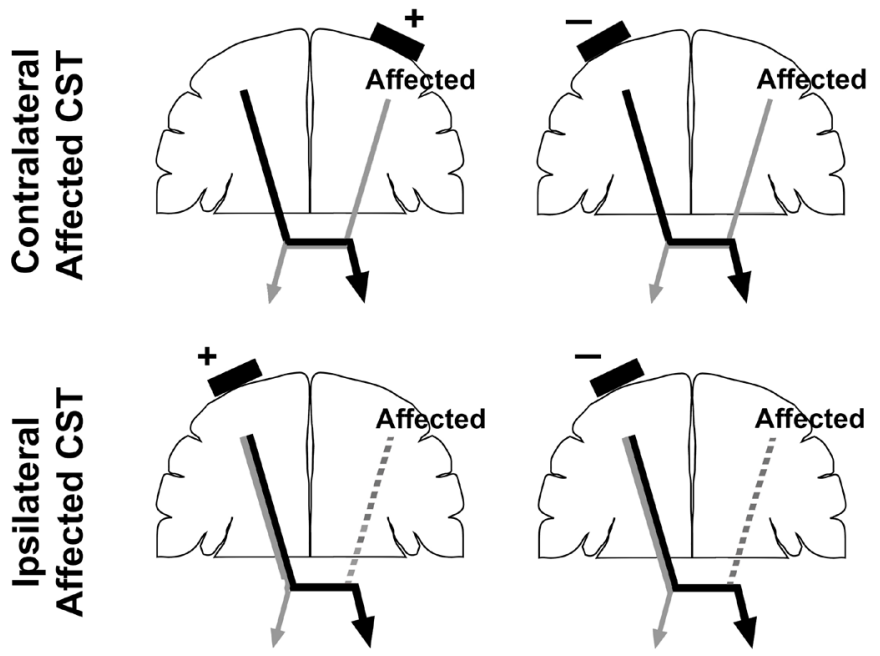

Figure 1 CST connectivity patterns and tDCS montages. Top row: CST connectivity is maintained from lesioned hemisphere to affected hand. Targeted tDCS: anode placed over motor map of affected UE in affected hemisphere. Untargeted tDCS: cathode placed over lessaffected hemisphere. Bottom row: CST connectivity is lost from lesioned hemisphere, and shifted to the ipsilateral hemisphere. Targeted tDCS: anode placed over motor map of affected UE in less affected hemisphere. Untargeted tDCS: cathode placed over less affected hemisphere. For all tDCS montages, the second electrode will be placed on the forehead contralateral to the first electrode. CST, corticospinal tract; tDCS, transcranial direct current stimulation; UE, upper extremity.

connections to the affected UE will show the most robust changes in motor cortex physiology after therapy, which will correlate with changes in hand function.

\section{METHODS AND ANALYSIS}

\section{Public/patient involvement statement}

Pilotdatawere collected on 20 children (age $=10.8 \pm 2.6$ years, range $=7-18$ years $)$. Parents and participants provided ongoing daily feedback on the feasibility and acceptability of tDCS. Two study personnel have cerebral palsy and have been instrumental in the design of the study from its conception.

\section{Overall study design}

We will conduct a single-blinded randomised controlled trial (RCT) with stratification based on CST connectivity, age and gender to determine whether efficacy of HABIT $+\mathrm{tDCS}$ depends on the targeting of tDCS to the locus of motor control of the impaired UE. We will compare three types of tDCS: (1) anodal tDCS targeted to either the affected UE motor map, (2) cathodal tDCS targeted to the unaffected UE motor map and (3) sham tDCS. In all groups, tDCS will be paired with HABIT (10 hours: 2 hours/day for 5 days) (figure 2 ). 


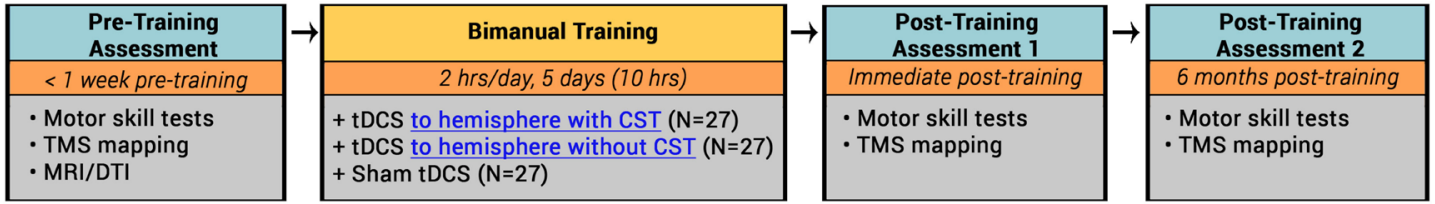

Figure 2 Experimental design. tDCS, transcranial direct current stimulation; TMS, transcranial magnetic stimulation.

\section{Participants}

Eighty-one children with USCP, aged 6-17 years, will be enrolled (figure 2). Participants will be recruited by advertising at our respective institutions, mailings, local clinics/hospitals, our existing database of more than 800 eligible individuals and social media.

When a child and their family express interest in enrolment, we will send them a health survey via a HIPAAsecure, NIH-supported clinical database (REDCap). The primary exclusion criteria (table 1) pertain to risks associated with MRI, single-pulse transcranial magnetic stimulation (TMS) and tDCS. We will discuss the study, risks and the child's health history in detail with families to confirm eligibility. Eligible children and caregivers will be invited to our facilities for consent, review of study and testing and intervention by the study coordinator.

\section{Number of participants}

The primary clinical outcomes are Box and Blocks Test (BB) for unimanual dexterity and the Assisting Hand Assessment (AHA) for bimanual function. The estimated effect size from previous studies ${ }^{141518}$ and pilot data is estimated to be 0.35 (BB change $3, \mathrm{SD}=10.2$ blocks, $\mathrm{AHA}$ change 2, $\mathrm{SD}=9.3 \mathrm{AHA}$ units, $\alpha=0.05$ (two-tailed) and $\beta=0.8$ ). We estimate that 22 subjects will be needed per group. We will recruit $20 \%$ more children than needed for the primary analyses, to account for children whose affected UE is controlled by both hemispheres and dropouts. Thus, 81 children (27 children/group) will be recruited.

\section{Randomisation procedure}

We will use a computer-generated blocked randomisation stratified by CST connectivity (determined with TMS), age and gender with concealed allocation for prospective allocation to the three groups: targeted tDCS, untargeted tDCS and sham tDCS. We will follow intention-to-treat principles. Randomisation occurs after baseline assessments to allow CST connectivity determination.

\section{Blinding}

Children and their families, study personnel, clinical evaluators and AHA scorers will be blinded to treatment (active vs sham tDCS) allocation. One principal investigator (PI) will not be blinded, as it is critical for one person to monitor the quality and settings of the tDCS devices, to optimise safety and protocol adherence and fidelity. TMS and diffusion tensor imaging (DTI) data analysis will be performed using coded file names.

\section{General methods}

\section{Bimanual training}

All participants will receive HABIT for 2 hours/day on 5 consecutive days ( 10 hours). This duration was chosen as changes in most clinical outcomes following bimanual training alone have been shown to require at least 30 hours, ${ }^{19} 20$ and thus the dose will be subthreshold such that findings would not be washed out with the addition of tDCS. Nonetheless, our pilot data suggests that children will improve on goal performance (Canadian Occupational Performance Measure, COPM) even with HABIT alone (sham), thus providing some potential benefits for all participants. Participants will not receive other treatments during the 5 days of treatment but may continue ongoing care during the 6 month of follow-up. HABIT will be conducted at either Teachers College (TC), Columbia University, New York, New York, USA, or Burke Neurological Institute (BNI), White Plains, New York, USA. HABIT relies on principles of motor learning and plasticity ${ }^{21-28}$ and largely parallels constraint-induced movement therapy. ${ }^{2628}{ }^{29}$ Each child will be assigned to an interventionist to maintain at least a 1:1 ratio. Children can work individually with their interventionist or with other children. Our study, funded before the COVID-19 pandemic began, proposed to conduct the intervention in groups of four-six children to allow for social interaction, peer-modelling and encouragement. Given ongoing COVID-19 precautions, we may need to reduce our group size to two-three children (plus each child's interventionist and supervisors), spread children out across rooms and limit and/or maintain social distancing for group activities. Example activities for HABIT $+\mathrm{tDCS}$ include playing cards, building with blocks, throwing and catching a ball, arts and crafts, and functional tasks such as buttoning. ${ }^{21}$

\section{Task selection}

We have identified age-appropriate fine and gross motor activities that require use of both hands. ${ }^{21}$ Activities are selected by considering the role of the involved limb increasing in complexity from passive assist to active manipulator. Both positive reinforcement and knowledge of results provide motivation and reinforce target movements. ${ }^{30}$ Instructions are given to the child before the start of each task reminding children how each hand will be used during the activity, ${ }^{31}$ although problem-solving is highly encouraged.

Whole task practice involves performing repetitive practice of targeted movements embedded in a play activity. An example is a card game. The motor components of 
Table 1 Inclusion and exclusion criteria

\begin{tabular}{l} 
Criterion \\
\hline Inclusion criteria \\
Age: $6-17$ years \\
Diagnosis of unilateral CP \\
Parent/guardian willing to provide \\
informed consent \\
Participant willing to provide \\
informed assent \\
Ability to pick up, hold and release \\
a light object with affected hand \\
Exclusion criteria \\
Current medical illness unrelated \\
to CP \\
$\begin{array}{l}\text { Seizure beyond } 2 \text { years of age, } \\
\text { use of anti-seizure medication, } \\
\text { history of epilepsy, cranial metal } \\
\text { implants, structural brain lesion, } \\
\text { devices that may be affected } \\
\text { by tDCS or TMS (pacemaker, } \\
\text { medication pump, cochlear } \\
\text { implant and implanted brain } \\
\text { stimulator) }\end{array}$ \\
\hline
\end{tabular}

\section{Method of ascertainment}

Medical records

Physical health screening and examination of neurological reports

Meeting with principal investigator (PI) to discuss Required

study and sign consent form

Meeting with $\mathrm{PI}$ to discuss study, signing assent Required form in the presence of $\mathrm{PI}$

Pre-intervention screening measures and score under the maximum Jebsen-Taylor Test of Hand Function (JTTHF) ceiling of 1080s

Medical history and physical examination

Medical records, interview with participant and parent(s), and use of a checklist

\section{Intervention may be too challenging for the child}

\section{Justification}

Children $<6$ years of age may have difficulty tolerating procedures and may have small head size

Target population of the trial

May impair child's ability to comply with trial and may affect study results

TMS and tDCS may increase risk of seizure in subjects prone to seizures

Cognitive deficits

High motor ability in affected arm

\section{Severe spasticity \\ Lack of asymmetry in hand function}

Pre-intervention screening measures; Kaufman Brief Intelligence Test, score \pm 1 SD from normal

JTTHF score of $<50 \%$ differences between the two hands or score $<100$ secs

Pre-intervention screening measures; Modified Ashworth test score $>3$ (>moderate)

Pre-intervention screening measures; JTTHF score of $<50 \%$ differences between the two hands

Orthopaedic surgery in affected arm in last 12 months

Botulinum toxin therapy in either UE during last 6 months, or planned during the study period

Currently receiving intrathecal baclofen

\section{True positive response on the} TMS and tDCS safety screen

Current use of medications known Medical records and interview with participant to lower the seizure threshold

\section{Previous episode of unprovoked} neurocardiogenic syncope

Indwelling metal or incompatible medical devices

\section{Centrally acting medications,} including anti-seizure medications

Evidence of scalp disease or skin abnormalities
Medical records, interview with participant and parent(s)

Medical records and interview with participant and parent(s)

Medical records and interview with participant and parent(s)

Interview with participant and parent(s) and parent(s)

Medical records and interview with participant and parent(s)

Medical records and interview with participant and parent(s)

Medical records and interview with participant and parent(s)

Medical records and interview with participant and parent(s)
Child needs to understand study assent and instructions related to the testing and intervention

Child may not benefit from interventions due to mild hand function or extremely limited use of the affected hand

May confound ability to drive changes in motor control quality

May suggest bilateral CP

Recovery may confound study results

Change in tone may confound study results

Change in tone may confound study results

Would indicate an increased risk of seizure

Underlying condition may pose risk of seizure and medication may influence TMS results

Could be exacerbated by TMS

Metallic objects in body may shift during MRI, posing risk of injury

Underlying condition may pose risk of seizure and medication may influence TMS results

tDCS may exacerbate the skin condition or increase discomfort

CP, cerebral palsy; tDCS, transcranial direct current stimulation; TMS, transcranial magnetic stimulation; UE, upper extremity. 
play involve holding cards in one hand, and picking up and placing down cards with the other hand.

Part task practice involves isolating a single component of the activity and performing it repeatedly. For example, after playing a card game, the child may be asked to flip cards over simultaneously with each hand, using a supination movement, as quickly as possible. The interventionist records the number of cards the child can turn in $30 \mathrm{~s}$ and the procedure is repeated several times.

\section{Grading task difficulty}

Depending on the child's motor capabilities and designated target movements, playing can be structured to grade the difficulty of a specific movement. Skill progression, ${ }^{32}$ where we use part and whole practice to drive performance and scaffold the environment to facilitate success and grade difficulty, has been shown to be the essential ingredient to enhance performance ${ }^{32} 33$ and drive motor map expansion. ${ }^{18}$ These outcomes are independent of CST laterality. ${ }^{34}{ }^{35}$ In the context of a card game, cards can be placed farther away from the child to encourage elbow extension, or on an elevated surface, to encourage wrist extension.

\section{Training and supervision of intervention providers}

Interventionists are students in the kinesiology or neuroscience and education programme at TC and local universities. Interventionists are trained with a standardised protocol. Interventionists are supervised by experienced physical and occupational therapists to ensure consistent approaches are used and treatment adherence and fidelity are maintained. Throughout each session, the supervisor (who will train all interventionists and be present at both sites) will oversee each child's activities and progress, and will rotate through each participant-interventionist pair to provide modelling and feedback and ensure treatment fidelity. The supervisor and study PIs will meet daily with interventionists to discuss the progress of each child, problemsolve and serve to identify key goals for the following day. The high ratio of interventionist to child and supervisor to interventionist further enables treatment consistency, adherence and fidelity.

\section{Determination of CST laterality}

We will determine CST laterality in two ways. (1) TMS map side (primary measure): we will determine which hemisphere evokes movement of the affected hand when TMS is applied (within a latency of $40 \mathrm{~ms}$, to rule out indirect motor pathways). If both hemispheres elicit motor evoked potentials (MEPs) (bilateral CST connectivity), the side with the greatest area (\# of sites) will be used to denote the dominant side. (2) DTI (secondary measure): we will use DTI to visualise the affected CST in cases where laterality cannot be determined by TMS. There is high concordance between these approaches $(\mathrm{p}<0.001$, sensitivity $=93 \%$, specificity $=85 \%){ }^{36}$

\section{Transcranial direct current stimulation}

A $2 \mathrm{~mA}$ current will be delivered using surface rubbercarbon electrodes $\left(35 \mathrm{~cm}^{2}\right)$ embedded in saline soaked sponges $(0.9 \% \mathrm{NaCl})$ by a battery driven, constant current stimulator (Soterix LTE). A $2 \mathrm{~mA}$ tDCS has been shown to improve dexterity in typically developing children more than $1 \mathrm{~mA}$ without increased side effects. ${ }^{37}$ Participants randomised to receive tDCS will receive stimulation during the first $20 \mathrm{~min}$ of HABIT while seated, with the anode either over motor hotspot of the side containing CST connectivity for the first dorsal interosseous (FDI) of the more affected hand as identified using TMS (targeted tDCS) or the cathode over the contralesional hemisphere (untargeted tDCS, figure 1). For the targeted tDCS group, the anode will be placed over the motor hotspot of the side containing CST connectivity for the FDI as identified using TMS, and the cathode will be placed on the supraorbital area contralateral to the anode. The targeted hemisphere will depend on whether the affected CST has a contralateral or ipsilateral organisation pattern. For the untargeted tDCS group, the cathode will be placed over the contralesional motor cortex, and the anode will be placed on the supraorbital area contralateral to the cathode. A 20-minute duration overlapping physical training was chosen as it has been safely tested in several studies with children with USCP. ${ }^{5131637-39}$ For participants randomised to receive sham tDCS, a comparable preparation will be performed and will include a 30 -second real current ramping to $2 \mathrm{~mA}$ at commencement, followed by a $5 \mathrm{~s}$ slow decrease, with no current sustained during the 20 min. ${ }^{13} 3738$ We will record the amount of saline used and electrode contact quality (measured by the device). The tDCS will be performed by one study PI who is not blinded to the type of stimulation. This person will monitor contact quality and ensure fidelity is maintained. Study personnel will also measure blood pressure before and after tDCS, as well as any side effects using a post brain stimulation symptoms checklist (see online supplemental file).

\section{MEASURES OF HAND FUNCTION}

Assessments were chosen to capture changes in (1) unimanual dexterity, (2) bimanual performance and (3) functional use of the affected hand (figure 3 ). Tests will be performed and videoed by an evaluator blinded to the child's CST laterality and treatment group before, immediately after, and 6 months after treatment. The assessments will occur at the location HABIT was provided (BNI or TC) before, immediately and 6 months after treatment by the same assessor at each time point and caregivers will complete questionnaires during the child's evaluation.

Two primary outcome measures will quantify bimanual performance and unimanual capacity under the Activity domain of the International Classification of Functioning, Disability and Health (ICF). ${ }^{40}$ This domain is most relevant the targeted upper extremity function. 


\begin{tabular}{|c|c|c|c|c|}
\hline Assessment & Measurement & Outcome Measure & Test Duration & Equipment \\
\hline \multicolumn{5}{|c|}{ Primary Measures: } \\
\hline $\begin{array}{l}\text { Transcranial Magnetic } \\
\text { Stimulation }\end{array}$ & CST laterality & $\begin{array}{l}\text { Which hemisphere evokes movement of } \\
\text { the affected hand when TMS is applied }\end{array}$ & 90 minutes & $\begin{array}{l}\text { TMS device, Neuroconn } \\
\text { system, EMG electrodes }\end{array}$ \\
\hline Box and Blocks Test & $\begin{array}{c}\text { Unimanual } \\
\text { movement speed }\end{array}$ & $\begin{array}{l}\text { \# of } 2.5 \mathrm{~cm}^{3} \text { blocks moved from one box, } \\
\text { over a barrier, into adjacent box in } 1 \mathrm{~min}\end{array}$ & 30 minutes & $\begin{array}{c}\begin{array}{c}\text { BBT test kit - box, blocks, } \\
\text { blindfold, nitrile gloves }\end{array} \\
\end{array}$ \\
\hline $\begin{array}{l}\text { Assisting Hand } \\
\text { Assessment }\end{array}$ & Bimanual UE use & $\begin{array}{c}\text { Effectiveness with which child uses affected } \\
\text { UE in bimanual activities (AHA Units) }\end{array}$ & 20 minutes & $\begin{array}{c}\text { AHA test kit - } \\
\text { board game, toys }\end{array}$ \\
\hline \multicolumn{5}{|c|}{ Secondary Measures: } \\
\hline $\begin{array}{l}\text { Structural MRI, } \\
\text { Diffusion Tensor Imaging }\end{array}$ & CST laterality & $\begin{array}{l}\text { Which hemisphere shows } \\
\text { visualization of the affected CST }\end{array}$ & 1 hour & $\begin{array}{c}\text { MRI scanner, } \\
\text { DTI Studio software }\end{array}$ \\
\hline $\begin{array}{c}\text { Cooper } \\
\text { Stereognosis }\end{array}$ & $\begin{array}{c}\text { Ability to identify } \\
\text { objects using only touch }\end{array}$ & $\begin{array}{l}\text { \# of objects identified correctly (out of } \\
\text { 16), time taken to identify each object }\end{array}$ & 20 minutes & $\begin{array}{l}16 \text { standardized objects \& } \\
\text { shapes for child to identify }\end{array}$ \\
\hline ABILHAND-Kids & $\begin{array}{l}\text { UE impairments in } \\
\text { children }\end{array}$ & $\begin{array}{l}\text { Parent/caregiver report of child's ability } \\
\text { to perform } 21 \text { specific motor tasks }\end{array}$ & 5 minutes & $\begin{array}{l}\text { Questionnaire form } \\
\text { (parent/caregiver) }\end{array}$ \\
\hline $\begin{array}{l}\text { Canadian Occupational } \\
\text { Performance Measure }\end{array}$ & $\begin{array}{l}\text { Assess outcome relating } \\
\text { to goals for self-care }\end{array}$ & $\begin{array}{c}\text { Parent/caregiver report of child's performance } \\
\text { of up to } 5 \text { parent- or child-selected self-care } \\
\text { goals, as well as satisfaction }\end{array}$ & 5 minutes & $\begin{array}{l}\text { Questionnaire form } \\
\text { (parent/caregiver) }\end{array}$ \\
\hline $\begin{array}{l}\text { Participation and } \\
\text { Environment Measure- } \\
\text { Children and Youth }\end{array}$ & $\begin{array}{l}\text { Assess participation in } \\
\text { the home, school and } \\
\text { community settings }\end{array}$ & $\begin{array}{l}\text { Parent/caregiver report of child's } \\
\text { participation in } 3 \text { settings, alongside } \\
\text { environmental factors ( } 20 \text { questions) }\end{array}$ & 5 minutes & $\begin{array}{l}\text { Questionnaire form } \\
\text { (parent/caregiver) }\end{array}$ \\
\hline $\begin{array}{l}\text { Dimensions of Mastery } \\
\text { Questionnaire }\end{array}$ & $\begin{array}{l}\text { Assess child's mastery- } \\
\text { related behaviors }\end{array}$ & $\begin{array}{c}\text { Parent/caregiver report of child's levels of } \\
\text { mastery motivation (41 questions) }\end{array}$ & 5 minutes & $\begin{array}{l}\text { Questionnaire form } \\
\text { (parent/caregiver) }\end{array}$ \\
\hline
\end{tabular}

Figure 3 Assessments. AHA, Assisting Hand Assessment; BBT, Box and Blocks Test; CST, corticospinal tract; TMS, transcranial magnetic stimulation; UE, upper extremity. EMG, electromyography, DTI, diffusion tensor imaging.

1. AHA: the $\mathrm{AHA}^{41}{ }^{42}$ measures and describes the effectiveness with which a child with a unilateral disability makes use of his/her affected (assisting) hand in performance of bimanual activities. The AHA is conducted through scoring of observable performance skills exposed during meaningful occupational performance (play). AHA is a standardised and criterion referenced test for children with unimanual motor impairments, test validity for all items, $99 \% \mathrm{CI}^{41}$ and excellent reliability (0.97 interrater and 0.99 intrarater) ${ }^{43}$ It is sensitive to change in USCP. ${ }^{44} \mathrm{~A}$ functionally meaningful score change is 4 logit points. ${ }^{43}$

2. BB: children will sit at a table in front of rectangular box divided into two compartments. One compartment contains 150 wooden $2.5 \mathrm{~cm}^{3}$ blocks. ${ }^{45}$ Children will be asked to move blocks, one at a time, with one hand, from one compartment to the other. The number of blocks moved in $60 \mathrm{~s}$ is recorded for each hand (interrater reliability of 0.95 , reliable and responsive to change).$^{46}$ A functionally meaningful score change is 1.9 blocks on the more affected hand, 3.0 blocks on the less affected hand. ${ }^{46}$

Secondary measures will be used across the three ICF domains:

1. ABILHAND-Kids (ICF activity domain) measures the ability of a child to perform specific motor tasks, regardless of strategy. A caregiver completes the survey about the child's abilities. It has been validated for children with $\mathrm{CP}$, has a strong reliability $(\mathrm{R}=0.94)$ and reproducibility $(\mathrm{R}=0.91) .{ }^{47}$

2. COPM (ICF activity domain) was designed to identify and measure, by means of interview, changes in functional problems clients consider to be relevant in the areas of self-care, productivity and leisure performance. The client or caregiver defines the most relevant functional goals, ranks their importance and rates their child's performance and their own satisfaction level. ${ }^{4-50}$ It is valid and reliable for use with parents, ${ }^{50}$ and provides outcomes relevant to children and their families. ${ }^{51} 52$

3. Cooper Stereognosis ${ }^{53}$ (ICF body structure and function domain) measures the ability of a child to identify 16 small objects and shapes using only tactile input. The child will sit at a table, blindfolded. Objects will be placed individually and the child must feel the object with one hand and identify it. Each hand will be tested separately and the number of objects correctly identified is recorded. Its interrater reliability is $0.85 .^{53}$

4. Dimensions of Mastery Questionnaire ${ }^{54}$ (ICF activity domain) will be used to assess the level of motivation in mastering challenging tasks. The parent-report questionnaire assesses instrumental (persistence at objectrelated tasks, social activities with adults and peers, and gross motor tasks) and expressive (behavioural indicators of positive affect and negative reaction to failure) motivation. Subscale scores will be used to determine whether motivation impacts gains in functional motor skills. It has good reliability (0.84) and discriminant and concurrent validity are supported. ${ }^{55}$

5. Participation and Environment Measure-Children and Youth (PEM-CY) (ICF participation domain) evaluates participation in the home, school and in the community, alongside environmental factors within these settings. The PEM-CY can be used for children 5-17 years old, with or without disabilities. Internal consistency and reliability are moderate to good. ${ }^{56}$

\section{Expected outcome}

We predict that all groups will show improved goal performance, and that there will be a significant interaction between stimulation type and improvement in dexterity and quality of bimanual performance, with children receiving stimulation targeted to the motor map of the affected UE showing greater improvements than children receiving other tDCS conditions immediately after treatment and maintained at the 6-month follow-up. 
We will assess changes in motor cortex excitability measures using TMS associated with HABIT+tDCS.

\section{Transcranial magnetic stimulation}

TMS will be conducted at BNI for all participants making the process as child friendly as possible. ${ }^{35}$

\section{Resting motor threshold (rMT)}

Resting motor threshold (rMT) is a measure of excitability of the motor cortex. The rMT is the minimum stimulator output required to evoke an MEP over $50 \mu \mathrm{V}$ in the FDI muscle in 6 of 10 trials while children have relaxed their arms. ${ }^{57}$

\section{Active motor threshold (aMT)}

Active MT (aMT) is a measure of excitability of the motor cortex. The aMT is the minimum stimulator output required to evoke an FDI MEP over $50 \mu \mathrm{V}$ in 6 of 10 trials during low-level squeeze of the tips of the thumb and index finger. ${ }^{57}$

\section{Recruitment curve}

Recruitment curves (RCs) quantify changes in MEP over different stimulus intensities. Ten TMS pulses will be delivered at $<0.1 \mathrm{~Hz}$ at each of the following stimulation levels: $90 \%$ rMT, $110 \%$ rMT, $120 \%$ rMT, $130 \%$ rMT and $150 \%$ rMT. RCs will be done at hotspots for each FDI. Stimuli will be delivered in an order unpredictable to subjects. RCs will be performed up to $150 \%$ rMT or maximum stimulator output, whichever is lower.

\section{MEP amplitude}

MEP is a measure of the strength of motor response to TMS. Electromyography (EMG) recordings will be exported to MATLAB for filtering and processing. The MEP for each muscle at each stimulation site will be defined as the peak-to-peak amplitude of the EMG response. Trials will be excluded if the child was not relaxed before the TMS pulse. MEPs will be averaged for stimuli delivered at the same site.

\section{Bilateral TMS mapping of motor cortex}

Muscle activity will be recorded using surface EMG electrodes. A multichannel recording system (NeuroConn, Germany) will be used to simultaneously record EMG activity bilaterally in the FDI, wrist flexor and extensor muscles. The TMS device will trigger the recording system such that EMG activity is recorded at $4000 \mathrm{~Hz} 400 \mathrm{~ms}$ before and $400 \mathrm{~ms}$ after each TMS pulse is delivered. The position of each stimulation point over the scalp will be recorded in three-dimensional and overlaid on the child's MRI using neuronavigation software (Brainsight Frameless, Rogue Research, Montreal, Canada).

Stimulation will begin in a medial portion of the affected motor cortex (M1). The coil will be progressed laterally until an MEP for the affected FDI is obtained. If an MEP is found, a rectangular grid of 1-centimetre spaced sites will be generated in Brainsight, centred around the point of strongest activation of the affected
FDI ('hotspot'). This site will be marked for subsequent use of the tDCS. The coil will be moved along the grid M1 until responses are no longer found for any recorded muscles. Both hemispheres will be mapped.

\section{Area of motor map}

If the average MEP is greater than $50 \mu \mathrm{V}$ for a muscle at one site, that site will be categorised as controlling that muscle. The total number of sites will constitute the area of digit and wrist maps for each hand. This measure serves as the primary measure of motor cortical physiology.

\section{Expected outcome}

We predict that changes in motor map size and cortical excitability will be largest when tDCS is targeted to the cortex controlling movement of the affected hand. We further expect to have determined that changes will be larger in response to HABIT plus targeted tDCS compared with HABIT plus untargeted tDCS and HABIT plus sham tDCS.

\section{MRI}

Each child will undergo a structural MR scan and diffusion tensor (DT) imaging scan without sedation on a Siemens MRI tDCS study protocol at the Citigroup Biomedical Imaging Centre at Weill Cornell Medicine, New York, New York, USA. The structural MRI will be used to co-register TMS stimulation targets with specific brain landmarks for TMS neuronavigation. For TMS localisation, there is normal variability in brain topography relative to scalp landmarks. For structural scans, 165-slice images will be taken at a resolution of $256 \times 256$ pixels. The structural MRI will also be used to identify the lesion type and extent. The DTI scan will be performed during the same session using a 65-direction protocol, 75 slices per direction at a resolution of $112 \times 112$ pixels each.

\section{Classification of CP aetiology}

Each child's medical history (in particular the neurological report) will be used to determine their diagnosis, and this will be confirmed by the child's physical or occupational therapist and during the screening process.

\section{DT tractography}

DT images will be imported into DTI Studio software (V.3, Johns Hopkins University, Baltimore, Maryland, USA) for processing and analyses. Image series for each participant will be screened for movement artefact, and slices showing artefact will be removed. Since we will obtain images using 65 gradients and will perform duplicate scans, up to $30 \%$ of slices can be removed without compromising feasibility of tract reconstruction. After screening for movement artefact, colour maps of fractional anisotropy will be constructed, showing the integrity of different neural pathways. To visualise fibre streams, seeds will be placed in the internal capsule and cerebral peduncle. 


\section{Statistical analysis plans}

The accuracy of all data will be verified by two researchers. For both aims, a statistician blinded to treatment group will use a group $\times$ test session analysis of variance (ANOVA) with repeated measures on test session and Tukey post-hoc tests corrected for multiple comparisons to examine differences among groups on each primary and secondary measure of hand function. The interaction will determine differential group effects, whereas a lack of an interaction will indicate statistically similar outcomes irrespective of treatment group. If data are missing, we will use a mixed linear model analysis. We will also add in (stepwise) covariates, including gender, age, side of impairment, lesion type, lesion size, CST connectivity, CST fractional anisotropy and number of streamlines (determined by DTI), and baseline hand function. If the data are not normally distributed, we will use nonparametric statistics.

\section{Procedure for handling missing data}

Intention-to-treat analysis will be used. To account for children who miss assessments, we will analyse data using a mixed linear model regression, which accounts for unequal time points among individuals. Mixed linear models on test sessions will be performed for all clinical outcomes with time as a fixed factorial factor to see improvements over time. Mixed linear models allow the estimation of interindividual variability and intraindividual patterns of change over time, while accounting for missing data.

\section{Data management}

All data will be stored for 3 years after study completion. Data analysis will be conducted in collaboration with a statistician. Data will be stored on an online, HIPAAcompliant database (REDCap). All study-related electronic files will be accessible only by key personnel, and all computers will be password protected. All subjects will be given a unique identifier at the time of enrolment that will be used for all study-related documentation. Paper case report forms and study files will be kept in the study coordinator's locked cabinet in a secure office.

\section{Resource sharing plan}

We have made a commitment to publish, in a timely manner, all the relevant scientific information that they will derive during this project. Unpublished information could be made available to interested parties via a request to the principal investigator. Anonymised data will be made available via the Data and Specimen Hub at the Eunice Kennedy Shriver National Institute of Child Health and Development.

\section{ETHICS AND DISSEMINATION}

The study has been approved by the BRANY Institutional Review Board (IRB; \#18-10-285-512) and is registered with clinicaltrials.gov. The study will be conducted according to the principles of the Declaration of Helsinki. Safety will be monitored by an independent data safety monitoring board (DSMB), which will meet twice per year to monitor progress and safety. Subjects will be discontinued in the case of an adverse event. Important protocol modifications will be reported to the IRB, DSMB and clinicaltrials. gov. The results of this RCT will be published in open access, peer-reviewed scientific journals and presented at national and international meetings. We will leverage our patient and family relationships to maximise dissemination. The study results will be shared with the academic and stakeholder community, including dissemination of training tools through patient associations and patient/ family advocacy groups. Participants will receive a plain language report at the end of the study.

\section{Author affiliations}

${ }^{1}$ Department of Biobehavioral Science, Teachers College, Columbia University, New York, New York, USA

${ }^{2}$ Burke Neurological Institute, White Plains, New York, USA

${ }^{3}$ Department of Occupational Therapy, Boston University, Boston, Massachusetts, USA

${ }^{4}$ Department of Rehabilitation, Laval University, Quebec City, Quebec, Canada ${ }^{5}$ Department of Occupational Therapy, Universidade Federal de Minas Gerais, Belo Horizonte, Brazil

Acknowledgements We thank the children and families who participated in the pilot study and the volunteers who worked with the participants. We thank Bernadette Gillick for assistance with transcranial direct current stimulation application in children with unilateral spastic cerebral palsy and Talita Campos, Grace-Anne Herard, Lindsey Soles, Orit Herzberg-Keller, Ellen Osei, Genni Hester and Amanda Sarafian for clinical evaluations.

Contributors AMG and KMF are the study principal investigators, conceptualised the study concept and study design, are responsible for the conduct and reporting of the work, drafted the final version of this manuscript and will take the lead roles on preparation for publication of the clinical outcomes. CLF and MR assisted with collection and analysis of pilot data. KC was responsible for ethics applications and reporting and summarising preliminary data. MB analysed pilot AHA videos and supervised interventions. All authors have contributed to the writing and critical review of the manuscript and have approved the final version.

Funding This work is supported by the National Institutes of Health (NIH) (grant number: 2R01 HD076436-06) to KMF and AMG. MR was supported by a postdoctoral training award from Fonds de Recherche en Santé du Québec. CLF was supported by NIH/NCATS (grant number: \#TL1-TR-002386).

Competing interests None declared.

Patient consent for publication Consent obtained from parent(s)/guardian(s)

Provenance and peer review Not commissioned; externally peer reviewed.

Supplemental material This content has been supplied by the author(s). It has not been vetted by BMJ Publishing Group Limited (BMJ) and may not have been peer-reviewed. Any opinions or recommendations discussed are solely those of the author(s) and are not endorsed by BMJ. BMJ disclaims all liability and responsibility arising from any reliance placed on the content. Where the content includes any translated material, BMJ does not warrant the accuracy and reliability of the translations (including but not limited to local regulations, clinical guidelines, terminology, drug names and drug dosages), and is not responsible for any error and/or omissions arising from translation and adaptation or otherwise.

Open access This is an open access article distributed in accordance with the Creative Commons Attribution Non Commercial (CC BY-NC 4.0) license, which permits others to distribute, remix, adapt, build upon this work non-commercially, and license their derivative works on different terms, provided the original work is properly cited, appropriate credit is given, any changes made indicated, and the use is non-commercial. See: http://creativecommons.org/licenses/by-nc/4.0/.

ORCID iD

Andrew M Gordon http://orcid.org/0000-0003-1041-0479 


\section{REFERENCES}

1 Novak I, Morgan C, Fahey M, et al. State of the evidence traffic lights 2019: systematic review of interventions for preventing and treating children with cerebral palsy. Curr Neurol Neurosci Rep 2020;20:3.

2 Cada EA, O'Shea RK. Identifying barriers to occupational and physical therapy services for children with cerebral palsy. J Pediatr Rehabil Med 2008;1:127-35.

3 Majnemer A, Shevell M, Law M, et al. Participation and enjoyment of leisure activities in school-aged children with cerebral palsy. Dev Med Child Neurol 2008;50:751-8.

4 Shikako-Thomas K, Fehlings D, Germain M, et al. Current Practice "Constraints" in the Uptake and Use of Intensive Upper Extremity Training: A Canadian Perspective. Phys Occup Ther Pediatr 2018;38:143-56.

5 Gillick BT, Gordon AM, Feyma T, et al. Non-Invasive brain stimulation in children with unilateral cerebral palsy: a protocol and risk mitigation guide. Front Pediatr 2018;6:56.

6 Nitsche MA, Paulus W. Sustained excitability elevations induced by transcranial DC motor cortex stimulation in humans. Neurology 2001;57:1899-901.

7 Reis J, Schambra HM, Cohen LG, et al. Noninvasive cortical stimulation enhances motor skill acquisition over multiple days through an effect on consolidation. Proc Natl Acad Sci U S A 2009;106:1590-5.

8 Buch ER, Santarnecchi E, Antal A, et al. Effects of tDCS on motor learning and memory formation: a consensus and critical position paper. Clin Neurophysiol 2017:128:589-603.

9 Ciechanski P, Cheng A, Lopushinsky S, et al. Effects of transcranial direct-current stimulation on neurosurgical skill acquisition: a randomized controlled trial. World Neurosurg 2017:108:876-84.

10 Cole L, Giuffre A, Ciechanski P, et al. Effects of high-definition and conventional transcranial direct-current stimulation on motor learning in children. Front Neurosci 2018;12:787.

11 Elsner B, Kwakkel G, Kugler J, et al. Transcranial direct current stimulation (tDCS) for improving capacity in activities and arm function after stroke: a network meta-analysis of randomised controlled trials. J Neuroeng Rehabil 2017;14:95

$12 \mathrm{Xu} \mathrm{J}$, Branscheidt M, Schambra H, et al. Rethinking interhemispheric imbalance as a target for stroke neurorehabilitation. Ann Neurol 2019;85:502-13.

13 Kirton A, Ciechanski P, Zewdie E, et al. Transcranial direct current stimulation for children with perinatal stroke and hemiparesis. Neurology 2017:88:259-67.

14 Gillick B, Menk J, Mueller B, et al. Synergistic effect of combined transcranial direct current stimulation/constraint-induced movement therapy in children and young adults with hemiparesis: study protocol. BMC Pediatr 2015;15:178.

15 Gillick BT, Feyma T, Menk J, et al. Safety and feasibility of transcranial direct current stimulation in pediatric hemiparesis: randomized controlled preliminary study. Phys Ther 2015;95:337-49.

16 Rich TL, Nemanich S, Chen M, et al. Transcranial direct current stimulation (tDCS) paired with Occupation-Centered bimanual training in children with unilateral cerebral palsy: a preliminary study. Neural Plast 2018;2018:9610812.

17 Inguaggiato E, Bolognini N, Fiori S, et al. Transcranial direct current stimulation (tDCS) in unilateral cerebral palsy: a pilot study of motor effect. Neural Plast 2019;2019:2184398.

18 Friel KM, Kuo H-C, Fuller J, et al. Skilled bimanual training drives motor cortex plasticity in children with unilateral cerebral palsy. Neurorehabil Neural Repair 2016;30:834-44.

19 Sakzewski L, Provan K, Ziviani J, et al. Comparison of dosage of intensive upper limb therapy for children with unilateral cerebral palsy: how big should the therapy pill be? Res Dev Disabil 2015;37:9-16.

20 Brandão MB, Mancini MC, Ferre CL, et al. Does dosage matter? A pilot study of hand-arm bimanual intensive training (habit) dose and dosing schedule in children with unilateral cerebral palsy. Phys Occup Ther Pediatr 2018;38:227-42.

21 Charles J, Gordon AM. Development of hand-arm bimanual intensive training (habit) for improving bimanual coordination in children with hemiplegic cerebral palsy. Dev Med Child Neurol 2006;48:931-6.

22 Eliasson AC, Gordon AM. Constraint-induced movement therapy for children with hemiplegia. In: Eliasson AC, Burtner P, eds. Improving hand function in children with cerebral palsy. London: MacKeith Press, 2008: 308-19.

23 Gordon AM, Charles J, Wolf SL. Methods of constraint-induced movement therapy for children with hemiplegic cerebral palsy: development of a child-friendly intervention for improving upperextremity function. Arch Phys Med Rehabil 2005;86:837-44.

24 Gordon AM, Charles J, Wolf SL. Efficacy of constraint-induced movement therapy on involved upper-extremity use in children with hemiplegic cerebral palsy is not age-dependent. Pediatrics 2006;117:e363-73.

25 Brandão MB, Ferre C, Kuo H-C, et al. Comparison of structured skill and unstructured practice during intensive bimanual training in children with unilateral spastic cerebral palsy. Neurorehabil Neural Repair 2014;28:452-61.

26 Gordon AM, Schneider JA, Chinnan A, et al. Efficacy of a handarm bimanual intensive therapy (habit) in children with hemiplegic cerebral palsy: a randomized control trial. Dev Med Child Neurol 2007;49:830-8.

27 Gordon AM, Chinnan A, Gill S, et al. Both constraint-induced movement therapy and bimanual training lead to improved performance of upper extremity function in children with hemiplegia. Dev Med Child Neurol 2008;50:957-8.

28 Gordon AM, Hung Y-C, Brandao M, et al. Bimanual training and constraint-induced movement therapy in children with hemiplegic cerebral palsy: a randomized trial. Neurorehabil Neural Repair 2011;25:692-702.

29 Charles JR, Wolf SL, Schneider JA, et al. Efficacy of a child-friendly form of constraint-induced movement therapy in hemiplegic cerebral palsy: a randomized control trial. Dev Med Child Neurol 2006;48:635-42.

30 Robert MT, Sambasivan K, Levin MF. Extrinsic feedback and upper limb motor skill learning in typically-developing children and children with cerebral palsy: review. Restor Neurol Neurosci 2017;35:171-84.

31 Steenbergen B, Gordon AM. Activity limitation in hemiplegic cerebral palsy: evidence for disorders in motor planning. Dev Med Child Neurol 2006;48:780-3.

32 Brandão $\mathrm{MB}$, Ferre $\mathrm{C}$, Kuo $\mathrm{H}-\mathrm{C}$, et al. Comparison of structured skill and unstructured practice during intensive bimanual training in children with unilateral spastic cerebral palsy. Neurorehabil Neural Repair 2014;28:452-61.

33 Hung Y-C, Brandão MB, Gordon AM. Structured skill practice during intensive bimanual training leads to better trunk and arm control than unstructured practice in children with unilateral spastic cerebra palsy. Res Dev Disabil 2017;60:65-76.

34 Smorenburg ARP, Gordon AM, Kuo H-C, et al. Does corticospinal tract connectivity influence the response to intensive bimanual therapy in children with unilateral cerebral palsy? Neurorehabil Neural Repair 2017;31:250-60.

35 Friel K, Ferre CL, Brandao M, et al. Improvements in upper extremity function following intensive training are independent of corticospinal tract organization in children with unilateral spastic cerebral palsy: a clinical randomized trial. Frontiers Neurology. In Press.

36 Kuo H-C, Ferre CL, Carmel JB, et al. Using diffusion tensor imaging to identify corticospinal tract projection patterns in children with unilateral spastic cerebral palsy. Dev Med Child Neurol 2017:59:65-71.

37 Ciechanski P, Kirton A. Transcranial direct-current stimulation can enhance motor learning in children. Cereb Cortex 2017;27:2758-67.

38 Bikson M, Grossman P, Thomas C, et al. Safety of transcranial direct current stimulation: evidence based update 2016. Brain Stimul 2016:9:641-61.

39 Friel KM, Gordon AM, Carmel JB, et al. Pediatric Issues in Neuromodulation: Safety, Tolerability and Ethical Considerations. In: Kirton A, Gilbert D, eds. Pediatric brain stimulation. Oxford: Academic Press, 2016: 133-47.

40 Organization WH. International classification of functioning, disability, and health (ICF). Geneva: World Health Organization, 2001.

41 Krumlinde-Sundholm L, Eliasson AC. Development of the assisting hand assessment: a Rasch-built measure intended for children with unilateral upper limb impairments. , 2003: 10, 13.

42 Krumlinde-Sundholm L, Holmefur M, Kottorp A, et al. The assisting hand assessment: current evidence of validity, reliability, and responsiveness to change. Dev Med Child Neurol 2007;49:259-64.

43 Holmefur M, Krumlinde-Sundholm L, Eliasson A-C. Interrater and intrarater reliability of the assisting hand assessment. Am J Occup Ther 2007;61:79-84.

44 Eliasson A-C, Krumlinde-sundholm L, Shaw K, et al. Effects of constraint-induced movement therapy in young children with hemiplegic cerebral palsy: an adapted model. Dev Med Child Neurol 2005;47:266-75.

45 Mathiowetz V, Volland G, Kashman N, et al. Adult norms for the box and block test of manual dexterity. Am J Occup Ther 1985;39:386-91.

46 Araneda R, Ebner-Karestinos D, Paradis J, et al. Reliability and responsiveness of the Jebsen-Taylor Test of Hand Function and the Box and Block Test for children with cerebral palsy. Dev Med Child Neurol 2019:61:1182-1188. 
47 Arnould C, Penta M, Renders A, et al. ABILHAND-Kids: a measure of manual ability in children with cerebral palsy. Neurology 2004;63:1045-52.

48 de Brito Brandão M, Gordon AM, Mancini MC. Functional impact of constraint therapy and bimanual training in children with cerebral palsy: a randomized controlled trial. Am J Occup Ther 2012;66:672-81.

49 Law M, Baptiste S, McColl M, et al. The Canadian occupational performance measure: an outcome measure for occupational therapy. Can J Occup Ther 1990;57:82-7.

50 Verkerk GJQ, Wolf MJMAG, Louwers AM, et al. The reproducibility and validity of the Canadian occupational performance measure in parents of children with disabilities. Clin Rehabil 2006;20:980-8.

51 Cusick A, McIntyre S, Novak I, et al. A comparison of goal attainment scaling and the Canadian occupational performance measure for paediatric rehabilitation research. Pediatr Rehabil 2006;9:149-57.

52 Randall KE, McEwen IR. Writing patient-centered functional goals. Phys Ther 2000;80:1197-203.
53 Cooper J, Majnemer A, Rosenblatt B, et al. The determination of sensory deficits in children with hemiplegic cerebral palsy. J Child Neurol 1995;10:300-9.

54 Morgan G, Wang J, Xu Q. Using the Dimensions of Mastery Questionnaire to assess mastery motivation of English- and Chinese-speaking children: Psychometrics and implications for self regulation. In: Barrett K, ed. Handbook of self-regulatory processes in development: new directions and international perspectives. New York: Routledge/Taylor and Francis, 2013: 305-36.

55 Miller L, Marnane K, Ziviani J, et al. The dimensions of mastery questionnaire in school-aged children with congenital hemiplegia: test-retest reproducibility and parent-child concordance. Phys Occup Ther Pediatr 2014;34:168-84.

56 Coster W, Bedell G, Law M, et al. Psychometric evaluation of the participation and environment measure for children and youth. Dev Med Child Neurol 2011;53:1030-7.

57 Kleim JA, Kleim ED, Cramer SC. Systematic assessment of training-induced changes in corticospinal output to hand using frameless stereotaxic transcranial magnetic stimulation. Nat Protoc 2007;2:1675-84. 